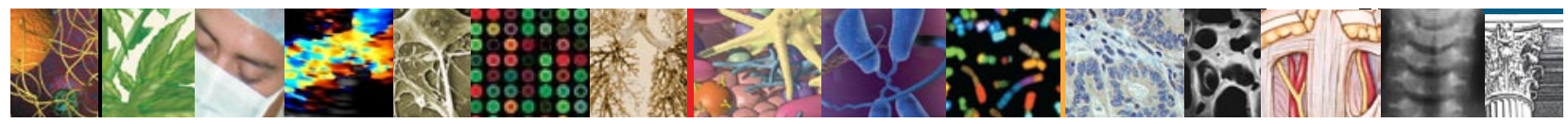 The NEW ENGLAND JOURNAL of MEDICINE
}

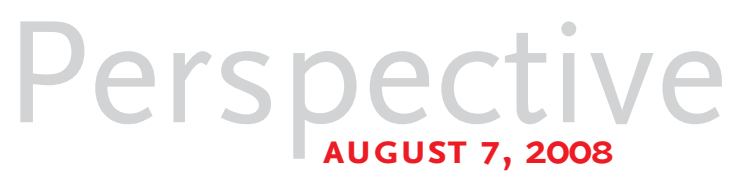

\section{The President's Emergency Plan for AIDS Relief - Is the Emergency Over?}

Wafaa M. El-Sadr, M.D., M.P.H., and David Hoos, M.D., M.P.H.

\section{Tn his 2003 State of the Union Address, President George W. Bush asked Congress to commit $\$ 15$ billion over the next 5 years for the President's Emergency Plan for AIDS Relief (PEPFAR) to com-}

bat the global epidemic of human immunodeficiency virus (HIV) infection. Thus was launched one of the largest international healthassistance programs in history. PEPFAR has since been both condemned as unilateral, paternalistic, narrowly focused, and distorted by a political agenda and lauded as groundbreaking, visionary, effective, and responsible for saving hundreds of thousands of lives. This year, Congress has had to consider the reauthorization of the program. On July 16, the Senate approved legislation that would increase the funding to $\$ 48$ billion for the next 5 years, sending the measure into conference committee. Thus, this seems an appropriate time to examine PEPFAR's achievements, limitations, and lessons for the future.

The HIV epidemic had been raging for more than two decades before the establishment of PEPFAR. For much of this time, the millions of HIV-infected people in resource-limited countries had little access to effective treatment. Instead, the response had focused almost exclusively on behaviorchanging strategies to prevent HIV transmission. Yet in wealthier countries, antiretroviral treatment was becoming increasingly effective in reducing morbidity and mortality, and in 2004, the global gap in access to treatment was starkly highlighted at the Inter- national AIDS Conference in Bangkok. At a time when only 50,000 Africans (1\% of the estimated 4.4 million in need) were receiving antiretroviral drugs, the conference's theme of "Access for All" became the rallying cry for the global HIV community. ${ }^{1}$ PEPFAR was part of the response to this emergency, meant to confront the epidemic that was causing indescribable suffering and destroying the fabric of communities around the world.

Since its inception, PEPFAR has faced criticism. Its most vocal critics have focused on some of its prevention strategies, which they view as driven by ideology rather than science. These include the overemphasis on abstinence as a key approach to risk reduction, the prohibition on support for commercial sex workers, and the opposition to needle and syringe exchange for injection-drug users. 


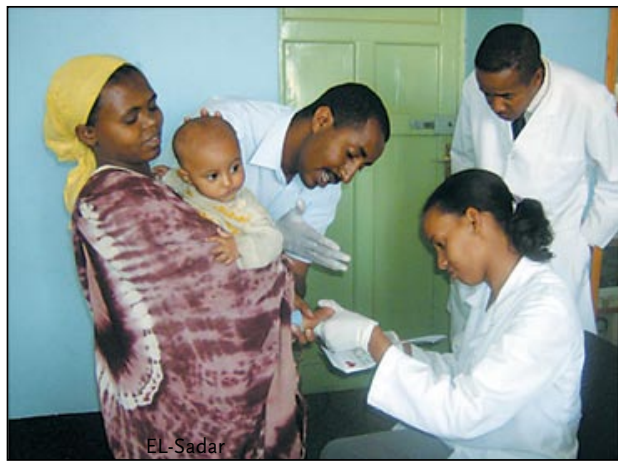

Obtaining a Blood Sample for HIV Testing, Gondar University Hospital, Gondar City, Northern Ethiopia.

But critics have also questioned PEPFAR's focus on HIV care and treatment. From the beginning, concerns have been raised regarding the feasibility and wisdom of expanding HIV treatment widely in resource-limited countries. Treatment was thought to be too complex, requiring management by physicians, who are scarce in these countries. For example, in Mozambique, which has an HIV prevalence of $16 \%$ among adults and an annual per capita health expenditure of $\$ 47$, there are only 3 physicians per 100,000 inhabitants. ${ }^{2}$ It was also feared that crumbling health care facilities would fail to cope with a greatly increased demand for services and that the absence of functioning laboratories would jeopardize the lives of patients who did receive treatment. Weak systems for pharmaceutical and commodity procurement with histories of gaps in availability were considered unlikely to deliver a secure drug supply. Some feared that reliance on generic antiretroviral drugs would lead to the use of low-quality knockoffs. Stigma was thought to present an insurmountable barrier that would inhibit patients from seeking treatment. It was also assumed that the requisite level of adherence to therapy was unlikely to be attained by poor people who had little experience with longterm medical care. Critics painted vivid images of drug-resistant HIV strains raging first through Africa and then throughout the world.

PEPFAR was also criticized for creating a vertical program with disease-specific goals, as well as a single-donor-driven structure and strategy, which some observers saw as threatening to undermine both national AIDS-control programs and the multilateral, multidonor Global Fund to Fight AIDS, Tuberculosis, and Malaria.

Given these reservations, how well has the program done? As of the end of March 2008, as a result of contributions by PEPFAR, a total of 1.7 million people had received antiretroviral therapy ${ }^{3}-$ reflecting an expansion of access to treatment by a factor of more than 10 in less than 4 years.

Despite its verticality, the program has also been reasonably well integrated with the global and national responses to the HIV epidemic. PEPFAR has collaborated with national AIDS-control programs in endorsing the approach of the World Health Organization, which recommends that each country have a single national HIV strategy, a single national treatment guideline, and a single framework for monitoring and evaluation. In most of the 15 countries where PEPFAR has focused its efforts, donors and host governments participate in multisectoral planning to ensure that donor funding complements the national AIDS-control strategy.

This effort has often revitalized national AIDS responses and may inspire similar coordination in re- lation to other health and development programs. Increasingly, PEPFAR funding is also supporting HIV treatment services beyond those provided by referral hospitals, including at district hospitals and primary health care centers, where most people receive care and where HIV services are by necessity integrated into primary care services.

The effect on the wider health care system of funding a diseasespecific program is harder to quantify. HIV disease, a chronic condition requiring follow-up during periods of health and illness, has motivated the establishment of systems to ensure continuity of care. Such systems are needed for the effective management of other chronic conditions, such as diabetes, cardiovascular disease, and mental illness. Funding from PEPFAR has contributed to enhanced support for pharmacies and laboratories and has spurred substantial growth of communitybased patient-support programs - services that may benefit broad populations of patients. Moreover, it has promoted the empowerment of persons living with HIV through the support of peereducation programs led by HIVinfected persons, which may have positive implications for creating health care systems that pay attention to the needs and priorities of patients and draw on their insights into program design and implementation. ${ }^{4}$ The types of programs eligible for PEPFAR funding have expanded to include services such as those for tuberculosis and malaria, two conditions highly relevant to HIV disease. There has also been support for the training of a wide array of health care providers, which may 
bring broad benefits to health care systems.

Despite PEPFAR's achievements, however, some critics argue that the program has fallen short in fundamental ways: it has not substantially altered the landscape in terms of stigma against people with HIV, inequity between the sexes, environmental threats to health, lack of educational opportunities for young people, and policies that restrict the expansion of the health care workforce. Such concerns suggest that it may be time for PEPFAR to transform itself from an emergency plan largely focused on preventing and treating HIV infection to one that aims to strengthen health care systems in general and to address root causes of the HIV epidemic, including social and economic factors.

In a sense, then, the key choice for PEPFAR at this crossroads is between a "pull" strategy and a "push" strategy: Will a continued singular focus on HIV "pull" other components of national health care systems forward, so that they become more responsive to other health threats and can catalyze the necessary changes in social norms and policy? Or will a "push" strategy that is focused primarily on strengthening health care systems and tackling fundamental issues that define societal vulnerabilities to disease be more effective in achieving both HIVspecific and broader health and social goals?

Historically, strategies for controlling epidemics have ranged from treating diseases and preventing infections to addressing the environmental, social, attitudinal, and policy-related factors fueling the epidemic. An analysis of the history of epidemics in New York City, for example, noted that the decrease in their occurrence during the latter part of the 19th century was largely due to the implementation of fundamental changes in the city environment, combined with new public health policies. $^{5}$

However, these approaches may be erroneously perceived as dichotomous. Epidemics first require rapid action and concerted efforts to alleviate suffering making PEPFAR's early prioritization of treatment appropriate and humane. At this point in its evolution, there are loud calls for PEPFAR to pay greater attention to supporting overall health care systems and to addressing societal vulnerabilities to HIV. Yet the emergency is far from over. Despite heroic efforts, only $37 \%$ of the estimated number of people in need of treatment in the 15 PEPFAR focus countries are receiving such treatment. ${ }^{3}$ Although efforts to address underlying systemic problems are legitimate, intensive work is still necessary to expeditiously reach millions of people with HIV infection, and considerable work is needed to achieve HIV prevention goals.

The key challenge for PEPFAR will be to maintain its sense of urgency and its razor-sharp focus on results - factors that have resulted in remarkable achieve- ments in the face of enormous challenges. The advances have been dramatic, but much remains to be done.

Drs. El-Sadr and Hoos report receiving grant support through the Centers for Disease Control and Prevention and the U.S. Agency for International Development, including PEPFAR funds. No other potential conflict of interest relevant to this article was reported.

This article (10.1056/NEJMp0803762) was published at www.nejm.org on July 30 , 2008.

An interview with Dr. El-Sadr can be heard at www.nejm.org.

Dr. El-Sadr is director of the International Center for AIDS Care and Treatment Programs (ICAP) and a professor of clinical medicine and epidemiology at the Mailman School of Public Health and the College of Physicians and Surgeons, Columbia University, and chief of the Division of Infectious Diseases at Harlem Hospital Center - both in New York. Dr. Hoos is director of the Multicountry Columbia Antiretroviral Program at ICAP and assistant professor of clinical epidemiology at the Mailman School of Public Health.

1. UNAIDS. Progress report on the global response to the HIV/AIDS epidemic, 2003. (Accessed July 18, 2008, at http://data. unaids.org/Topics/UNGASS2003/ungass_ report_2003_en.pdf.)

2. Core health indicators: Mozambique. Geneva: World Health Organization, 2004. (Accessed July 18, 2008, at http://www. who.int/whosis/database/core/core_select_ process.cfm?country=moz\&indicators $=$ healthpersonnel.)

3. President's Emergency Plan for AIDS Relief. Latest PEPFAR treatment results. (Accessed July 6, 2008, at http://www.pepfar. gov.)

4. El-Sadr WM, Abrams EJ. Scale-up of HIV care and treatment: can it transform healthcare services in resource-limited settings? AIDS 2007;21:Suppl 5:S65-S70.

5. Rosner D. Hives of sickness: public health and epidemics in New York City. New Brunswick, NJ: Rutgers University Press, 1995.

Copyright $(2008$ Massachusetts Medical Society. 